\title{
ENERGETIC FERMI/LAT GRB100414A: ENERGETIC AND CORRELATIONS
}

\author{
Yuji Urata* \\ Institute of Astronomy and Astrophysics, Academia Sinica, PO BOX 23-141, Taipei 106, Taiwan \\ E-mail: uratadastro.ncu.edu.tw
}

\section{Kuiyun Huang}

Institute of Astronomy and Astrophysics, Academia Sinica, PO BOX 23-141, Taipei 106, Taiwan

\section{Kazutaka Yamaoka}

Department of Physics and Mathematics, Aoyama Gakuin University, 5-10-1, Fuchinobe,

Sayamihara 229-8558, Japan

\section{Makoto S. Tashiro}

Department of Physics, Saitama University, Shimo-Okubo, Saitama, 338-8570, Japan

\begin{abstract}
We present multi-wavelength observational results for energetic GRB 100414A with GeV photons. The prompt spectral fitting using Suzaku/WAM data yielded the spectral peak energies of $E_{\text {peak }}^{\text {src }}$ of $1458.7_{-106.6}^{+132.6} \mathrm{keV}$ and $E_{\text {iso }}$ of $34.5_{-1.8}^{+2.0} \times 10^{52} \mathrm{erg}$ with $z=1.368$. The optical afterglow light curves between 3 and 7 days were well fitted by a simple power law with a temporal index of $\alpha=-2.6 \pm 0.1$. The joint light curve with earlier Swift/UVOT observations yields a temporal break at $2.3 \pm 0.2$ days. This was the first Fermi/LAT detected event that demonstrated the clear temporal break in the optical afterglow. The jet opening angle derived from this temporal break was $\sim 5.8^{\circ}$, consistent with those of other well-observed long GRBs. Our multi-wavelength analyses showed that GRB $100414 \mathrm{~A}$ follows $E_{\text {peak }}^{\text {src }}-E_{\text {iso }}$ and $E_{\text {peak }}^{\text {src }}-E_{\gamma}$ correlations. The late afterglow revealed a flatter evolution with the significant excesses at 27.2 days. The most straightforward explanation for the excess is that GRB 100414A was accompanied by a contemporaneous supernova. The model light curve based on other GRB-SNe events is marginally consistent with the observed one.
\end{abstract}

Gamma-Ray Bursts 2012 Conference-GRB2012,

May 07-11, 2012

Munich, Germany

\footnotetext{
*This work was supporte by NSC 99-2112-M-001-002-MY3 and NSC 98-2112-M-008-003-MY3
} 


\section{Prompt emission}

GRB 100414A triggered the Suzaku WAM on 2010 April 14 02:20:22.879(=T0). The WAM [1] is a lateral shield of the Hard X-ray Detector on board the Suzaku satellite and can function as a GRB monitor sensitive to the 50-5000 keV gamma-rays (e.g. 2,3). The duration $T_{90}$ was $21.2 \pm 0.1$ $\mathrm{s}$ which places it in the class of long duration events. We performed the spectral analysis using the WAM transient data with 55 energy channels and 1-s time resolution. The WAM2 spectrum was extracted by integrating over T0-1.5 s to T0+24.5 s with the hxdmkwamspec in the standard HEADAS 6.9 package provided by NASA/GSFC. The background spectrum was estimated by incorporating the best-fit model of source-free time region on both sides (T0-504.5 to T0-4.5 and $\mathrm{T} 0+37.5$ to $\mathrm{T} 0+237.5)$ of the burst time region. We performed the spectral fitting in Xspec version 12.5 and found that the best-fit model was the GRB Band function $\left(\chi^{2} /\right.$ dof $\left.=24.4 / 24\right)$. Two other models we also attempted were a power-law $\left(\chi^{2} / \mathrm{dof}=253.5 / 26\right)$ and a power-law with an exponential cutoff $\left(\chi^{2} /\right.$ dof $\left.=35.3 / 25\right)$. The best-fit parameters for the Band function were $\alpha=-$ $0.62(-0.15,+0.17), \beta=-3.05(-0.28,+0.20)$, and $E_{\text {peak }}=616 \pm 34 \mathrm{keV}$. Thus obtained, the fluence in the $100-5000 \mathrm{keV}$ range was $(6.65 \pm 0.21) \times 10^{-5} \mathrm{erg} \mathrm{cm}^{-2}$. The corresponding Fermi/LAT photon data were downloaded from the Fermi Science Support Center. Based on the likelihood and aperture photometry, we generated the light curve for the $>100 \mathrm{MeV}$ energy range as shown in Figure 1 (left). The highest energy photon from this event was $\sim 4.3 \mathrm{GeV}$ at $\sim 40 \mathrm{~s}$ after the burst.
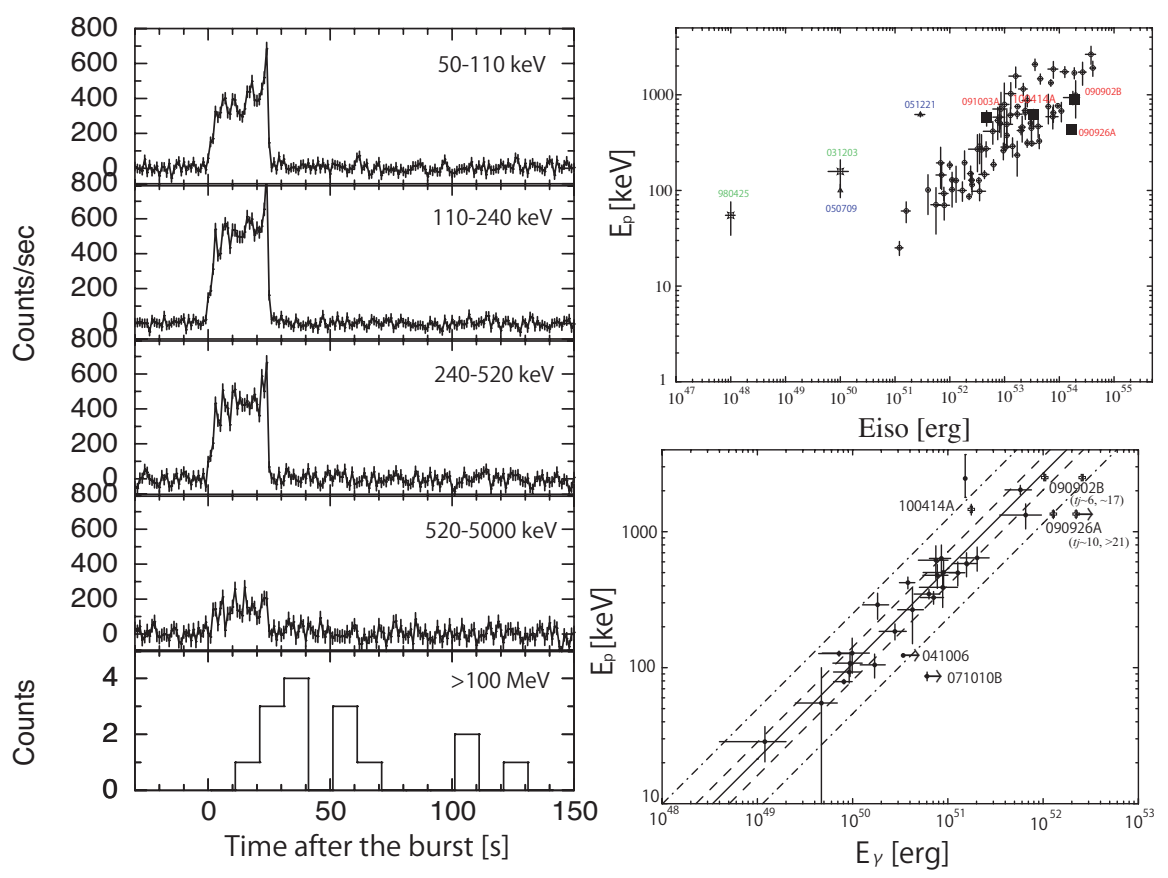

Figure 1: Left: Prompt X-ray and gamma-ray light curves of GRB100414A, as observed using Suzaku/WAM and Fermi/LAT. Top right: The $E_{\text {peak }}^{\text {src }}-E_{\text {iso }}$ relation combined with the data points of GRBs 100414A, 091003A, 090926A and 090902B (filled square). Bottom right: The $E_{\text {peak }}^{\text {src }}-E_{\gamma}$ relation including the data for GRBs 100414A, 090926A and 090902B corrected for a homogeneous circumburst medium. For GRBs 090926A and 090902B, there are two points for their possible jet break summarized in Table 1 . The dashed and dash-dotted lines indicate the $1 \sigma$ and $3 \sigma$ scatter of the correlation, respectively. 
Table 1: Suzaku/WAM observations of Fermi/LAT events with redshift.

\begin{tabular}{cccccccc}
\hline \hline GRB & $z$ & $\mathrm{~T}_{90}[\mathrm{~s}]$ & $\mathrm{E}_{\text {peak }}[\mathrm{keV}]$ & $t_{\text {jet }}[\mathrm{d}]$ & $E_{\text {iso }}\left[10^{52} \mathrm{erg}\right]$ & $E_{\gamma}\left[10^{50} \mathrm{erg}\right]$ & $E \mathrm{~h}[\mathrm{GeV}]$ \\
\hline $100414 \mathrm{~A}$ & 1.368 & 21 & $616_{-45}^{+56}$ & $2.3 \pm 0.2$ & $34.5_{-1.8}^{+2.0}$ & 17.6 & $4.3(40 \mathrm{~s})$ \\
091003 & 0.8969 & 23 & $576_{-72}^{+106}$ & - & $4.7_{-0.4}^{+0.6}$ & - & - \\
$090926 \mathrm{~A}$ & 2.1062 & 13 & $434_{-30}^{+32}$ & $\sim 10$ or $>21$ & $167.3_{-8.4}^{+11.9}$ & 128 or $>222$ & $19.6(25 \mathrm{~s})$ \\
$090902 \mathrm{~B}$ & 1.822 & 19 & $885_{-38}^{+39}$ & $\sim 6$ or $\sim 17$ & $193.3_{-3.2}^{+5.9}$ & 104 or 257 & $33.4(82 \mathrm{~s})$ \\
090510 & 0.903 & 0.33 & - & - & - & - & $30.5(0.829 \mathrm{~s})$ \\
\hline
\end{tabular}

\section{Optical Afterglow and Jet Break}

We performed the optical afterglow observations using the Canada France Hawaii Telescope (CFHT)/MegaCam within the framework of the EAFON (e.g. 4,5,6,7). The $r^{\prime}$-band monitoring observations covered the duration from 5.2 days to 57.6 days after the burst. Additional $g^{\prime}$ - and $i^{\prime}$-band images were taken on the night of 2010 April 20 (6.2 days after the burst). Because of the bright moon phase, there was no optical observation between 7 and 26 days. The standard CFHT's data pipeline reduced all of the data. The light curves demonstrated the steep decay occurring between 3 and 7 days after the burst. The temporal indices were $-2.3,-2.6 \pm 0.1$ and -2.7 in $g^{\prime}-, r^{\prime}$-, and $i^{\prime}$-bands, respectively. The spectral energy distribution (SED) of optical afterglow during the steep decay phase (at $\sim 6.2$ days) could be satisfactorily expressed according to a simple power law with the spectral index $\beta=1.2 \pm 0.2$. Despite the paucity of observations for the optical afterglow, the joint light curve using Swift/UVOT strongly suggested a temporal break. The decay indices before and after the break were $\sim 1$ and $\sim 2$, respectively, fully consistent with typical wellobserved long GRB optical afterglows. To examine whether the jet model is applicable to present afterglow, we utilized the relations (e.g [8]) and calculated $p$ and $\alpha$ based on the observed $\beta$. The observed values were in agreement with the calculated value of $\alpha=2.4 \pm 0.4$, assuming that we were observing a jet expansion phase in the frequency range above the synchrotron cooling. In addition, although the X-ray afterglow observation was also poor, the decay index $\alpha_{\mathrm{X}}=2.3 \pm 0.4$ between 2.0 and 7.4 days was also consistent with the jet model. The case of wind density profile with $v_{m}<v_{\text {opt }}<v_{X}<v_{c}$ also satisfied the closure relation. However, it is unlikely for the late (6.2 days) afterglow. We also attempted to fit the $g^{\prime}$-band light curve including the UVOT data using the broken power-law model by fixing the temporal decay indices of former and post jet break at -1.3 and -2.6 , respectively. The UVOT data were calibrated according to the SDSS field stars that have similar colors to the GRB afterglow, and were converted to $g^{\prime}$ band magnitudes. We successfully fitted the light curve and obtained the jet break time as $t=2.3 \pm 0.2$ days. The jet opening angle derived from this temporal break was $\sim 5^{\circ} .8$, consistent with those of other well-observed long GRBs (e.g. 9 and references therein).

\section{Energetic and Correlation}

The abundance of the multi-wavelength data for estimating the jet break time makes GRB 100414A one of the most favorable targets for evaluating the $E_{\text {peak }}^{\text {src }}-E_{\text {iso }}$ and $E_{\text {peak }}^{\text {src }}-E_{\gamma}$ relations of studies 
that had stagnated because of a lack of data on the $E_{\text {peak }}^{\text {src }}$ estimation and long-term optical monitoring. In particular, the current event was the first Fermi /LAT detected event exhibiting the clear jet break features. To examine the correlations with the least systematics errors, we collected five LAT-detected events observed using the Suzaku/WAM, as summarized in Table 1. The spectral analysis of the WAM data was performed in the same manner as described in Section 2.1. The estimated $E_{\text {iso }}$ of GRB 090902B in the $1 \mathrm{keV}$ to $10 \mathrm{MeV}$ range using Suzaku/WAM are consistent with the value derived from the Fermi /GBM observation[10]. In the case of GRB 090926A, the value is slightly smaller than that of the estimation $\left(2.3 \times 10^{54} \mathrm{erg}\right)$ using Fermi /GBM [11]. The most likely reason for this inconsistency is that the energy range of Suzaku/WAM is insufficient in detecting the underlying power-law component in the Fermi /LAT-GBM spectrum[12]. GRB 090510 was the short event, and the spectral parameters were not effectively constrained, we excluded this event for the evaluation of correlation. The marginal optical temporal break time $t_{\text {jet }}$ of GRB 090926A and GRB 090902B was reported $[10,11,13,14]$. As summarized in Table 1, the values vary widely according to the estimation methods. We also excluded the GRB 091003 for the evaluation of the $E_{\text {peak }}^{\text {src }}-E_{\gamma}$ relation because of a lack of the $t_{\text {jet }}$ estimation. Interestingly, the three selected events (GRB 090926A, GRB 090902B and the current one) shared commonality in the delayed highest energy photons from their main burst (GRB 090926A, GRB 090902B).

Figure 1 (Right) shows the $E_{\text {peak }}^{\text {src }}-E_{\text {iso }}$ and $E_{\text {peak }}^{\text {src }}-E_{\gamma}$ relations with Suzaku/WAM observed LAT-detected events. We found that all four events were highly consistent with previous studies of the $E_{\text {peak }}^{\text {src }}-E_{\text {iso. }}$ As shown in Figure 1, the measured values of current event $\left(E_{\text {peak }}^{\text {src }}=1458.7_{-106.6}^{+132.6}\right.$ $\mathrm{keV}$ and $E_{\text {iso }}=34.5_{-1.8}^{+2.0} \times 10^{52} \mathrm{erg}$ ) adhered closely to the $E_{\text {peak }}^{\text {src }}-E_{\text {iso }}$ relation. Although the background GRB physic of this correlation is not yet completely understood, the characteristics of different sub-classes of GRBs become apparent [15] . To compare other categories of the events (e.g., short duration GRBs and sub-energetic events), we plotted them in Figure 1. These features were clearly different from those of the current event. The present event and GRB 090902B were also consistent with $E_{\text {peak }}^{\text {src }}-E_{\gamma}$ correlation (Figure 1). In the case of GRB 090926A, $E_{\gamma}$ thoroughly depends on the jet break time estimation.

\section{References}

[1] Yamaoka, K., et al. Design and In-Orbit Performance of the Suzaku Wide-Band All-Sky Monitor, PASJ 61 (2009) 35

[2] Urata, Y., et al. Swifts GRB GRB071010B : outlier of the $\mathrm{E}_{\text {peak }}^{\mathrm{src}}-\mathrm{E}_{\gamma}$ and $\mathrm{E}_{\mathrm{iso}}-\mathrm{E}_{\text {peak }}^{\mathrm{src}}-\mathrm{t}_{\text {jet }}^{\mathrm{src}}$ correlations, Apj 706 (2009) L183

[3] Tashiro, M. S., et al. Swift and Suzaku Observations of the X-Ray Afterglow from the GRB 060105, PASJ 59 (2007) 361

[4] Urata, Y., et al. Multiband Optical Follow-up Observations of GRB 020813 at the Kiso and Bisei Observatories, ApJ 595 (2003) L21

[5] Urata, Y., et al. Testing the External-Shock Model of Gamma-Ray Bursts Using the Late-Time Simultaneous Optical and X-Ray Afterglows, ApJ 668 (2007) L95

[6] Huang, K.Y., et al. Optical Afterglow Observations of the Unusual Short-Duration Gamma-Ray Burst GRB 040924, ApJ 628 (2005) L93

[7] Huang, K.Y., et al. Multicolor Shallow Decay and Chromatic Breaks in the GRB 050319 Optical Afterglow, ApJ $6 \mathbf{6 5 4}$ (2007) L25 
[8] Sari, R., Piran, T., \& Halpern, J. P. Jets in Gamma-Ray Bursts, ApJ 519 (1999) L17

[9] Frail, D., et al. 2001 562, Beaming in Gamma-Ray Bursts: Evidence for a Standard Energy Reservoir, ApJ 562 (2001) L55

[10] Cenko, S. B., et al. Afterglow Observations of Fermi Large Area Telescope Gamma-ray Bursts and the Emerging Class of Hyper-energetic Events, ApJ 732 (2011) 29

[11] McBreen, S., et al. Optical and near-infrared follow-up observations of four Fermi/LAT GRBs: redshifts, afterglows, energetics, and host galaxies, A\&A 516 (2010) 71

[12] Abdo, A. A., et al. Fermi Observations of GRB 090902B: A Distinct Spectral Component in the Prompt and Delayed Emission, ApJ 706 (2009) L138

[13] Rau, A., et al. A Very Metal-poor Damped Lyman-Î́s System Revealed Through the Most Energetic GRB 090926A, ApJ 720 (2010) 862

[14] Swenson, C. A., et al. GRB 090926A and Bright Late-time Fermi Large Area Telescope Gamma-ray Burst Afterglows, ApJ 718 (2010) L14

[15] Amati, L. The $E_{\mathrm{p}, \mathrm{i}}-E_{\mathrm{iso}}$ correlation in GRBs: updated observational status, re-analysis and main implications, MNRAS 372 (2006) 233

[16] Urata, Y., et al. Very Early Multicolor Observations of the Plateau Phase of the GRB 041006 Afterglow, ApJ 655 (2007) L81

[17] Urata, Y., et al. Energetic Fermi/LAT GRB 100414A: Energetic and Correlations, ApJ 748 (2012) L4 